Supporting Inforamtion for:

\title{
An Uncatalyzed Meerwein-Ponndorf-Oppenauer-Verley Reduction of Aldehydes and Ketones Under Supercritical Conditions
}

\author{
Lena Sominsky, Esther Rozental, Hugo Gottlieb Aharon Gedanken* and Shmaryahu Hoz*
}

\author{
Gaussian archive files for structures appearing in Table 6.
}

\author{
i-PrOH

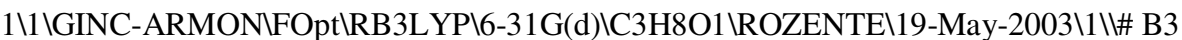 \\ LYP/6-31G* OPT=Z-MATRIX NOSYMM $\backslash C H 3-C H-O H-C H 3 \backslash \backslash 0,1 \backslash C \backslash C, 1, R 2 \backslash C, 2, R 3,1, A$ \\ 3\H,1,R4,2,A4,3,D4,0ไH,1,R5,2,A5,4,D5,0ไH,1,R6,2,A6,4,D6,0\O,2,R7,1,A7 \\ ,3,D7,0ไH,7,R8,2,A8,3,D8,0ไH,3,R9,2,A9,1,D9,0\H,3,R10,2,A10,8,D10,0\H,

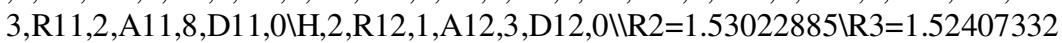 \\ $\backslash \mathrm{R} 4=1.09801025 \backslash \mathrm{R} 5=1.09591096 \backslash \mathrm{R} 6=1.09636485 \backslash \mathrm{R} 7=1.43096731 \mathrm{VR} 8=0.96989881$ \\ $\backslash \mathrm{R} 9=1.09447423 \backslash \mathrm{R} 10=1.09542488 \backslash \mathrm{R} 11=1.09589595 \backslash \mathrm{R} 12=1.10386167 \backslash \mathrm{A} 3=112.548$ \\ $88573 \backslash \mathrm{A} 4=111.0465583 \backslash \mathrm{A} 5=110.21826456 \backslash \mathrm{A} 6=111.24866655 \backslash \mathrm{A} 7=111.1201136 \backslash \mathrm{A} 8$ \\ $=107.56909077 \backslash \mathrm{A} 9=110.2740459 \backslash \mathrm{A} 10=110.83535215 \backslash \mathrm{A} 11=110.21222188 \backslash \mathrm{A} 12=108$ \\ $.71801664 \backslash \mathrm{D} 4=180.78212425 \backslash \mathrm{D} 5=119.76353417 \backslash \mathrm{D} 6=-119.73950332 \backslash \mathrm{D} 7=118.9633$ \\ 6638\D8=-180.0681154\D9=178.43681303\D10=177.02391534\D11=-62.70582443 \\ ID12=-120.34549548॥Version=IBM-RS6000-G98RevA.7\HF=-194.3533105\RMSD= \\ 4.063e-09\RMSF=1.946e-05\Dipole=-0.2611825,-0.3890042,-0.3953765\PG=C0 \\ $1[\mathrm{X}(\mathrm{C} 3 \mathrm{H} 8 \mathrm{O} 1)] \backslash 1 @$

\section{acetone}

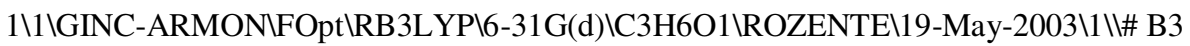 \\ LYP/6-31G* OPT=Z-MATRIX NOSYMM $\backslash \backslash \mathrm{CH} 3-\mathrm{C}=\mathrm{O}-\mathrm{CH} 3 \backslash \backslash 0,1 \backslash \mathrm{C} \backslash \mathrm{C}, 1, \mathrm{R} 2 \backslash \mathrm{C}, 2, \mathrm{R} 3,1, \mathrm{~A} 3 \backslash$ \\ H, 1,R4,2,A4,3,D4,0\H,1,R5,2,A5,4,D5,0\H,1,R6,2,A6,4,D6,0\O,2,R7,1,A7,3

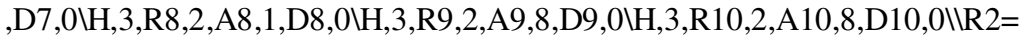 \\ $1.52045652 \backslash \mathrm{R} 3=1.52035998 \backslash \mathrm{R} 4=1.09177791 \backslash \mathrm{R} 5=1.09739445 \backslash \mathrm{R} 6=1.09742573 \backslash \mathrm{R} 7=$ \\ $1.21573261 \backslash \mathrm{R} 8=1.09177857 \backslash \mathrm{R} 9=1.09742232 \backslash \mathrm{R} 10=1.09741331 \backslash \mathrm{A} 3=116.53508581 \backslash$ \\ A4=109.83418362\A5=110.53766326\A6=110.51448118\A7=121.73801823\A8=109 \\ $.83984054 \backslash \mathrm{A} 9=110.53898137 \backslash \mathrm{A} 10=110.53507759 \backslash \mathrm{D} 4=180.10815218 \backslash \mathrm{D} 5=121.0286$ \\ 9553ไD6=-120.99397422\D7=180.0207613ไD8=180.03418852\D9=121.01043968\D \\ $10=-121.00805411 \|$ Version=IBM-RS6000-G98RevA.7 $\backslash \mathrm{HF}=-193.1556937 \backslash \mathrm{RMSD}=2$. \\ 738e-09\RMSF=5.066e-05\Dipole=0.9422745,0.0000456,-0.5828123\PG=C01 [X \\ (C3H6O1)]川@
}

\section{EtOH}

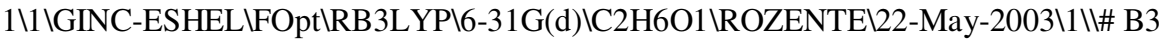
LYP/6-31G* OPT=Z-MATRIX NOSYMM $\backslash E$ thanol $\backslash 0,1 \backslash C \backslash C, 1, R 2 \backslash H, 2, R 3,1, A 3 \backslash H, 2$, R4,1,A4,3,D4,0ไH,1,R5,2,A5,3,D5,0ไH,1,R6,2,A6,3,D6,0ไH,1,R7,2,A7,3,D7, $0 \backslash \mathrm{O}, 2, \mathrm{R} 8,1, \mathrm{~A} 8,3, \mathrm{D} 8,0 \backslash \mathrm{H}, 8, \mathrm{R} 9,2, \mathrm{~A} 9,1, \mathrm{D} 9,0 \backslash \mathrm{R} 2=1.52629544 \backslash \mathrm{R} 3=1.10256452 \backslash \mathrm{R}$ $4=1.09499813 \backslash \mathrm{R} 5=1.09501774 \backslash \mathrm{R} 6=1.09812096 \backslash \mathrm{R} 7=1.09669193 \backslash \mathrm{R} 8=1.42390463 \backslash \mathrm{R}$ $9=0.96994188 \backslash \mathrm{A} 3=110.15973375 \backslash \mathrm{A} 4=110.23991102 \backslash \mathrm{A} 5=110.45719863 \backslash \mathrm{A} 6=111.03$ $990201 \backslash \mathrm{A} 7=111.01580632 \backslash \mathrm{A} 8=112.84753308 \backslash \mathrm{A} 9=107.40625328 \backslash \mathrm{D} 4=117.91872139$ $\backslash \mathrm{D} 5=181.30316642 \backslash \mathrm{D} 6=61.42967789 \backslash \mathrm{D} 7=-58.1907704 \backslash \mathrm{D} 8=-124.73522051 \backslash \mathrm{D} 9=61$. 82195946।IVersion=IBM-RS6000-G98RevA.7\HF=-155.0342889\RMSD=1.367e-09 $\mathrm{RMSF}=1.920 \mathrm{e}-05 \backslash$ Dipole $=0.5099381,-0.0800525,-0.4086475 \backslash \mathrm{PG}=\mathrm{C} 01$ [X(C2H6O1 )$] \backslash @$

\section{MeCHO}

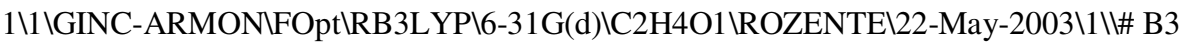


LYP/6-31G* OPT=Z-MATRIX NOSYMM \AAcytaldhyde $\backslash 10,1 \backslash C \backslash C, 1, R 2 \backslash O, 2, R 3,1, A 3 \backslash$

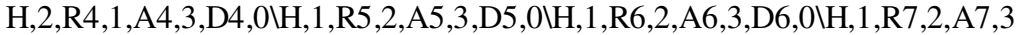

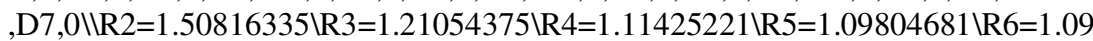
$232355 \backslash R 7=1.09805113 \backslash \mathrm{A} 3=124.69963901 \backslash \mathrm{A} 4=114.78991149 \backslash \mathrm{A} 5=109.80891123 \backslash \mathrm{A}$ $6=110.49342507 \backslash \mathrm{A} 7=109.80449045 \backslash \mathrm{D} 4=180.0815832 \backslash \mathrm{D} 5=121.47384601 \backslash \mathrm{D} 6=-0.01$ 747408\D7=-121.53143774IIVersion=IBM-RS6000-G98RevA.7\HF=-153.8301224\ $\mathrm{RMSD}=2.853 \mathrm{e}-09 \backslash \mathrm{RMSF}=4.809 \mathrm{e}-05 \backslash \mathrm{Dipole}=-0.7337936,-0.000191,-0.7355943 \backslash \mathrm{P}$ $\mathrm{G}=\mathrm{C} 01[\mathrm{X}(\mathrm{C} 2 \mathrm{H} 4 \mathrm{O} 1)] \| \backslash @$

\section{$\mathrm{PhCH}_{2} \mathrm{OH}$}

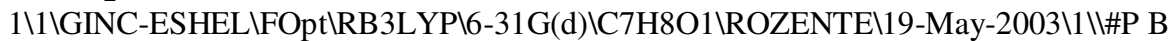
3LYP/6-31G* OPT=Z-MATRIX NOSYMM $\backslash \mathrm{Ph}-\mathrm{CH} 2-\mathrm{OH} \backslash \backslash 0,1 \backslash \mathrm{C} \backslash \mathrm{C}, 1, \mathrm{R} 2 \backslash \mathrm{C}, 2, \mathrm{R} 3,1, \mathrm{~A} 3 \backslash \mathrm{C}$ ,3,R4,2,A4,1,D4,0\C,4,R5,3,A5,2,D5,0\C,1,R6,2,A6,3,D6,0\C,1,R7,2,A7,3, D7,0ไH,2,R8,1,A8,6,D8,0ไH,3,R9,2,A9,1,D9,0ไH,4,R10,3,A10,2,D10,0ไH,5,R 11,4,A11,3,D11,0〈H,6,R12,1,A12,2,D12,0ไH,7,R13,1,A13,6,D13,0〈H,7,R14,1 ,A14,6,D14,0\O,7,R15,1,A15,2,D15,0\H,15,R16,7,A16,1,D16,0\\R2=1.399517 $54 \backslash \mathrm{R} 3=1.3959404 \backslash \mathrm{R} 4=1.39589265 \backslash \mathrm{R} 5=1.39686221 \backslash \mathrm{R} 6=1.40091759 \backslash \mathrm{R} 7=1.5170460$ $4 \backslash \mathrm{R} 8=1.08837688 \backslash \mathrm{R} 9=1.08697671 \backslash \mathrm{R} 10=1.08680305 \backslash \mathrm{R} 11=1.08708852 \backslash \mathrm{R} 12=1.0862$ $9431 \backslash \mathrm{R} 13=1.10205535 \backslash \mathrm{R} 14=1.0967507 \backslash \mathrm{R} 15=1.42295918 \backslash \mathrm{R} 16=0.97059563 \backslash \mathrm{A} 3=120$ $.68286659 \backslash \mathrm{A} 4=120.03551664 \backslash \mathrm{A} 5=119.6540885 \backslash \mathrm{A} 6=118.9127969 \backslash \mathrm{A} 7=120.7434975$ $3 \backslash \mathrm{A} 8=119.55217168 \backslash \mathrm{A} 9=119.83891794 \backslash \mathrm{A} 10=120.15907828 \backslash \mathrm{A} 11=120.01707797 \backslash \mathrm{A} 1$ $2=118.93619208 \backslash \mathrm{A} 13=109.63355982 \backslash \mathrm{A} 14=110.29689843 \backslash \mathrm{A} 15=113.69455371 \backslash \mathrm{A} 16=$ $107.16931251 \backslash \mathrm{D} 4=0.55223369 \backslash \mathrm{D} 5=-0.08950145 \backslash \mathrm{D} 6=-0.63274925 \backslash \mathrm{D} 7=-181.60353$ $595 \backslash \mathrm{D} 8=-180.76261163 \backslash \mathrm{D} 9=179.95116059 \backslash \mathrm{D} 10=-180.36322594 \backslash \mathrm{D} 11=179.8189056$ $5 \backslash \mathrm{D} 12=180.69266005 \backslash \mathrm{D} 13=-160.10486427 \backslash \mathrm{D} 14=82.58480788 \backslash \mathrm{D} 15=146.28431964 \backslash$ D16=-56.49287986॥Version=IBM-RS6000-G98RevA.7\HF=-346.7705987\RMSD=3. 397e-09\RMSF=2.345e-05\Dipole=0.1571004,0.2568665,0.5202195\PG=C01 [X( C7H8O1)]川@

\section{PhCHO}

1\1\GINC-ARMONIFOpt\RB3LYP\6-31G(d)\C7H6O1\ROZENTE\20-May-2003\1\\# B3 LYP/6-31G* OPT=Z-MATRIX NOSYMM $\backslash \backslash B$ enzaldhyde $\backslash \backslash 0,1 \backslash C \backslash C, 1, R 1 \backslash C, 2, R 2,1, A 1 \backslash$

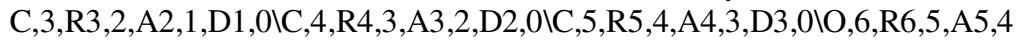

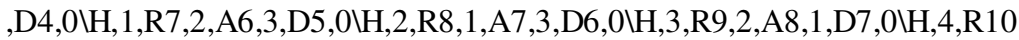
,3,A9,2,D8,0\C,5,R11,4,A10,3,D9,0\H,12,R12,5,A11,4,D10,0ไH,6,R13,5,A12

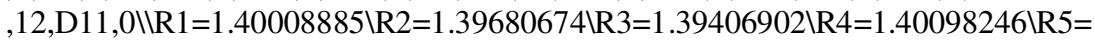
$1.48034784 \backslash \mathrm{R} 6=1.21630097 \backslash \mathrm{R} 7=1.08658225 \backslash \mathrm{R} 8=1.08687177 \backslash \mathrm{R} 9=1.0864423 \backslash \mathrm{R} 10=$ $1.08824345 \backslash \mathrm{R} 11=1.40338885 \backslash \mathrm{R} 12=1.08580652 \backslash \mathrm{R} 13=1.11325029 \backslash \mathrm{A} 1=120.3661486$ $7 \backslash \mathrm{A} 2=119.68352227 \backslash \mathrm{A} 3=120.17197006 \backslash \mathrm{A} 4=119.8228843 \backslash \mathrm{A} 5=124.81370504 \backslash \mathrm{A} 6=11$ $9.94535976 \backslash \mathrm{A} 7=119.82975981 \backslash \mathrm{A} 8=120.13542272 \backslash \mathrm{A} 9=120.33244519 \backslash \mathrm{A} 10=119.898$ $4294 \backslash \mathrm{A} 11=118.52074916 \backslash \mathrm{A} 12=114.55192343 \backslash \mathrm{D} 1=-0.00009518 \backslash \mathrm{D} 2=0.00025906 \backslash \mathrm{D} 3$ $=179.99974967 \backslash \mathrm{D} 4=179.99489345 \backslash \mathrm{D} 5=179.99979044 \backslash \mathrm{D} 6=-179.99981734 \backslash \mathrm{D} 7=-179$ $.9998532 \backslash \mathrm{D} 8=-179.99960199 \backslash \mathrm{D} 9=-0.00017951 \backslash \mathrm{D} 10=-179.99984183 \backslash \mathrm{D} 11=-179.99$ 287212l॥Version=IBM-RS6000-G98RevA.7 \HF=-345.5734557 \RMSD=5.517e-09\RM $\mathrm{SF}=4.968 \mathrm{e}-05 \backslash$ Dipole $=-0.654514,0.0000406,1.1228685 \mathrm{PG}=\mathrm{C} 01[\mathrm{X}(\mathrm{C} 7 \mathrm{H} 6 \mathrm{O} 1)] \backslash @$

\section{Cyclohexanol}

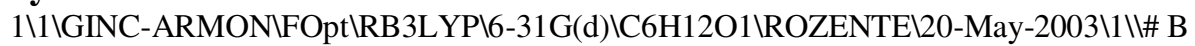
3LYP/6-31G* OPT=Z-MATRIX NOSYMM $\backslash$ Cyclohexanol $\backslash 0,1 \backslash \mathrm{ClC}, 1, \mathrm{R} 2 \backslash \mathrm{C}, 2, \mathrm{R} 3,1, \mathrm{~A}$ $3 \backslash \mathrm{C}, 3, \mathrm{R} 4,2, \mathrm{~A} 4,1, \mathrm{D} 4,0 \backslash \mathrm{C}, 4, \mathrm{R} 5,3, \mathrm{~A} 5,2, \mathrm{D} 5,0 \backslash \mathrm{C}, 1, \mathrm{R} 6,2, \mathrm{~A} 6,3, \mathrm{D} 6,0 \backslash \mathrm{H}, 3, \mathrm{R} 7,2, \mathrm{~A} 7$

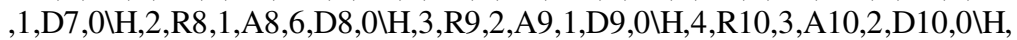
4,R11,3,A11,2,D11,0ไH,5,R12,4,A12,3,D12,0ไH,5,R13,4,A13,3,D13,0\O,1,R1 4,2,A14,3,D14,0〈H,6,R15,5,A15,4,D15,0ไH,6,R16,5,A16,4,D16,0〈H,2,R17,1,

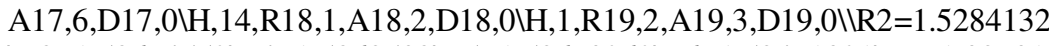
$\backslash \mathrm{R} 3=1.53674552 \backslash \mathrm{R} 4=1.5369589 \backslash \mathrm{R} 5=1.53670262 \backslash \mathrm{R} 6=1.53471021 \backslash \mathrm{R} 7=1.09701407 \backslash$ $\mathrm{R} 8=1.09641176 \backslash \mathrm{R} 9=1.10011294 \backslash \mathrm{R} 10=1.097391 \backslash \mathrm{R} 11=1.09983694 \backslash \mathrm{R} 12=1.0970701 \backslash$ $\mathrm{R} 13=1.10013175 \backslash \mathrm{R} 14=1.42756215 \backslash \mathrm{R} 15=1.10012965 \backslash \mathrm{R} 16=1.09898567 \backslash \mathrm{R} 17=1.0989$ $7113 \backslash \mathrm{R} 18=0.97018372 \backslash \mathrm{R} 19=1.10552873 \backslash \mathrm{A} 3=111.46097955 \backslash \mathrm{A} 4=111.52133499 \backslash \mathrm{A} 5=$ $111.3062653 \backslash \mathrm{A} 6=110.99554241 \backslash \mathrm{A} 7=109.88998176 \backslash \mathrm{A} 8=109.25512108 \backslash \mathrm{A} 9=109.393$ $77707 \backslash \mathrm{A} 10=110.29602885 \backslash \mathrm{A} 11=109.17149377 \backslash \mathrm{A} 12=110.50099493 \backslash \mathrm{A} 13=109.19089$ 
637\A14=107.14672045\A15=110.29598994\A16=110.08094014\A17=108.0217894 $7 \backslash \mathrm{A} 18=107.52577315 \backslash \mathrm{A} 19=108.3301345 \backslash \mathrm{D} 4=55.28908127 \backslash \mathrm{D} 5=-54.57631713 \backslash \mathrm{D} 6=-$ $55.4628132 \backslash \mathrm{D} 7=-182.01840678 \backslash \mathrm{D} 8=-178.59866002 \backslash \mathrm{D} 9=-65.58745468 \backslash \mathrm{D} 10=-177$. $22463042 \backslash \mathrm{D} 11=65.95365525 \backslash \mathrm{D} 12=-183.12014444 \backslash \mathrm{D} 13=-66.42444415 \backslash \mathrm{D} 14=-178.0$ $40722 \backslash \mathrm{D} 15=-177.44922786 \backslash \mathrm{D} 16=64.9100836 \backslash \mathrm{D} 17=65.53624143 \backslash \mathrm{D} 18=-180.903077$ $31 \backslash \mathrm{D} 19=63.52329486 \|$ Version=IBM-RS6000-G98RevA.7\HF=-311.0902461 $\backslash \mathrm{RMSD}=$ 6.699e-09\RMSF=4.061e-05 $\backslash$ Dipole $=0.5076587,0.0358017,-0.3418637 \backslash \mathrm{PG}=\mathrm{C} 01$ [X(C6H12O1)]川\@

\section{Cyclohexanone}

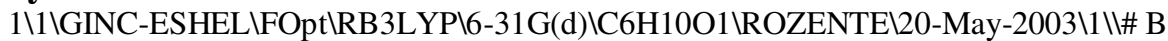
3LYP/6-31G* OPT=Z-MATRIX NOSYMM $\backslash C y c l o h e x a n o n e l \backslash 0,1 \backslash C \backslash C, 1, R 2 \backslash C, 2, R 3,1$,

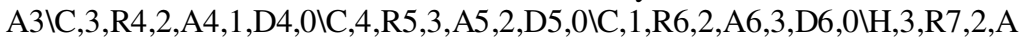

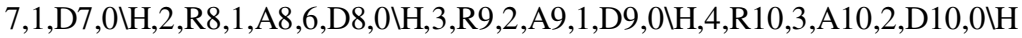

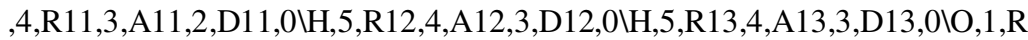
14,2,A14,3,D14,0\H,6,R15,5,A15,4,D15,0\H,6,R16,5,A16,4,D16,0ไH,2,R17,1

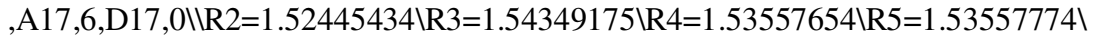
$\mathrm{R} 6=1.52463078 \backslash \mathrm{R} 7=1.09663648 \backslash \mathrm{R} 8=1.09397109 \backslash \mathrm{R} 9=1.09894967 \backslash \mathrm{R} 10=1.09700107$ $\backslash \mathrm{R} 11=1.10018627 \backslash \mathrm{R} 12=1.09663479 \backslash \mathrm{R} 13=1.09894248 \backslash \mathrm{R} 14=1.21640572 \backslash \mathrm{R} 15=1.093$ 95664\R16=1.10126766\R17=1.10122734\A3=111.85476888\A4=111.58620393\A5 $=111.13615823 \backslash \mathrm{A} 6=115.10712111 \backslash \mathrm{A} 7=109.60701023 \backslash \mathrm{A} 8=108.55424839 \backslash \mathrm{A} 9=109.0$ $7443306 \backslash \mathrm{A} 10=110.06485112 \backslash \mathrm{A} 11=109.4509984 \backslash \mathrm{A} 12=110.67162772 \backslash \mathrm{A} 13=109.2271$ $0509 \backslash \mathrm{A} 14=122.45540712 \backslash \mathrm{A} 15=112.1299356 \backslash \mathrm{A} 16=108.89419581 \backslash \mathrm{A} 17=107.5619046$

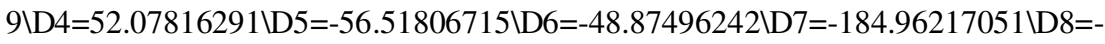
$173.17357309 \backslash \mathrm{D} 9=-68.68775011 \backslash \mathrm{D} 10=-178.71219656 \backslash \mathrm{D} 11=64.47303504 \backslash \mathrm{D} 12=-18$ $1.30099188 \backslash \mathrm{D} 13=-64.33807931 \backslash \mathrm{D} 14=-228.75194922 \backslash \mathrm{D} 15=-174.04263934 \backslash \mathrm{D} 16=66$ $.98332235 \backslash \mathrm{D} 17=70.62678727 \backslash \mathrm{V}$ ersion=IBM-RS6000-G98RevA.7\HF=-309.891291 $5 \backslash \mathrm{RMSD}=3.304 \mathrm{e}-09 \backslash \mathrm{RMSF}=3.836 \mathrm{e}-05 \backslash \mathrm{Dipole}=0.7225245,0.7212273,0.6476232 \backslash \mathrm{P}$ $\mathrm{G}=\mathrm{C} 01[\mathrm{X}(\mathrm{C} 6 \mathrm{H} 10 \mathrm{O} 1)] 1 \backslash @$

\section{$\operatorname{PhCH}(\mathrm{OH}) \mathrm{CH}_{3}$}

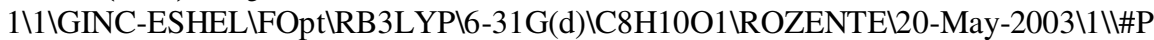
B3LYP/6-31G* OPT=Z-MATRIX NOSYMM $\backslash \mathrm{Ph}-\mathrm{CHCH} 3-\mathrm{OH} \backslash 10,1 \backslash \mathrm{C} \backslash \mathrm{C}, 1, \mathrm{R} 2 \backslash \mathrm{C}, 2, \mathrm{R} 3,1, \mathrm{~A}$ 3\C,3,R4,2,A4,1,D4,0\C,4,R5,3,A5,2,D5,0\C,1,R6,2,A6,3,D6,0\C,1,R7,2,A7

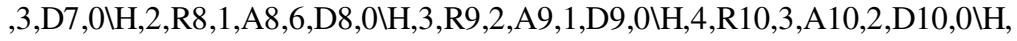

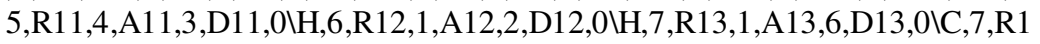
4,1,A14,6,D14,0\O,7,R15,1,A15,2,D15,0\H,15,R16,7,A16,1,D16,0\H,14,R17, 7,A17,1,D17,0ไH,14,R18,7,A18,1,D18,0ไH,14,R19,7,A19,1,D19,0\\2=1.3997 $7395 \backslash \mathrm{R} 3=1.39567118 \backslash \mathrm{R} 4=1.39613568 \backslash \mathrm{R} 5=1.39659224 \backslash \mathrm{R} 6=1.40098405 \backslash \mathrm{R} 7=1.5221$ $401 \backslash \mathrm{R} 8=1.08852251 \backslash \mathrm{R} 9=1.08701691 \backslash \mathrm{R} 10=1.08681281 \backslash \mathrm{R} 11=1.08713175 \backslash \mathrm{R} 12=1.08$ 631583\R13=1.10305669\R14=1.52919393\R15=1.42912193\R16=0.97057982\R17 $=1.09498735 \backslash \mathrm{R} 18=1.09494283 \backslash \mathrm{R} 19=1.09468608 \backslash \mathrm{A} 3=120.73689379 \backslash \mathrm{A} 4=120.03319$

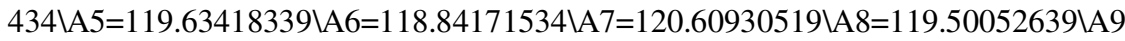
$=119.84605861 \backslash \mathrm{A} 10=120.16593293 \backslash \mathrm{A} 11=120.01967292 \backslash \mathrm{A} 12=118.95354552 \backslash \mathrm{A} 13=1$ $08.16761724 \backslash \mathrm{A} 14=112.284533 \backslash \mathrm{A} 15=112.15174191 \backslash \mathrm{A} 16=107.36949275 \backslash \mathrm{A} 17=110.1$ $4167161 \backslash \mathrm{A} 18=109.91677934 \backslash \mathrm{A} 19=110.6718787 \backslash \mathrm{D} 4=0.45192414 \backslash \mathrm{D} 5=-0.02839427 \backslash$ $\mathrm{D} 6=-0.59610025 \backslash \mathrm{D} 7=-181.76358753 \backslash \mathrm{D} 8=-180.7395956 \backslash \mathrm{D} 9=179.91345252 \backslash \mathrm{D} 10=-1$ $80.34820411 \backslash \mathrm{D} 11=179.71982308 \backslash \mathrm{D} 12=180.85606473 \backslash \mathrm{D} 13=-156.37520735 \backslash \mathrm{D} 14=84$ $.12952209 \backslash \mathrm{D} 15=146.02077742 \backslash \mathrm{D} 16=-53.64551663 \backslash \mathrm{D} 17=-59.8949593 \backslash \mathrm{D} 18=180.52$ 097054\D19=60.13258629 \Version=IBM-RS6000-G98RevA.7\HF=-386.089635\RM $\mathrm{SD}=7.368 \mathrm{e}-09 \backslash \mathrm{RMSF}=2.586 \mathrm{e}-05 \backslash \mathrm{Dipole}=0.1436868,0.2103939,0.4867366 \backslash \mathrm{PG}=\mathrm{C} 0$ $1[\mathrm{X}(\mathrm{C} 8 \mathrm{H} 10 \mathrm{O} 1)] / \backslash @$

\section{$\mathrm{PhCOCH}_{3}$}




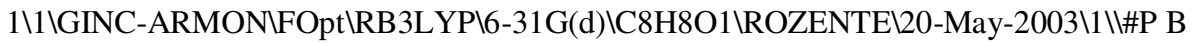

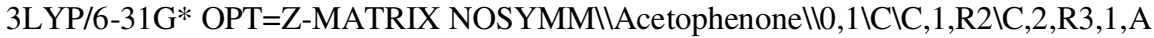
$3 \backslash \mathrm{C}, 3, \mathrm{R} 4,2, \mathrm{~A} 4,1, \mathrm{D} 4,0 \backslash \mathrm{C}, 4, \mathrm{R} 5,3, \mathrm{~A} 5,2, \mathrm{D} 5,0 \backslash \mathrm{C}, 1, \mathrm{R} 6,2, \mathrm{~A} 6,3, \mathrm{D} 6,0 \backslash \mathrm{C}, 1, \mathrm{R} 7,2, \mathrm{~A} 7$

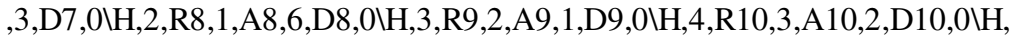

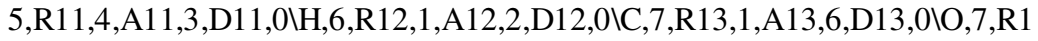
4,1,A14,2,D14,0〈H,13,R15,7,A15,1,D15,0ไH,13,R16,7,A16,1,D16,0ไH,13,R17

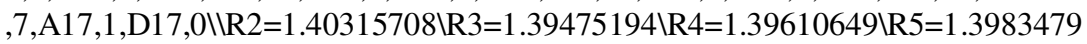

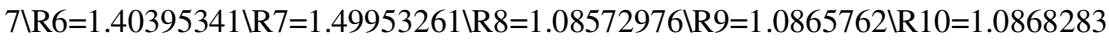
$2 \backslash \mathrm{R} 11=1.08666348 \backslash \mathrm{R} 12=1.08517514 \backslash \mathrm{R} 13=1.52001446 \backslash \mathrm{R} 14=1.22200797 \backslash \mathrm{R} 15=1.09$ $660955 \backslash \mathrm{R} 16=1.09132595 \backslash \mathrm{R} 17=1.09661982 \backslash \mathrm{A} 3=120.41390715 \backslash \mathrm{A} 4=120.00711746 \backslash \mathrm{A}$ $5=119.98002537 \backslash \mathrm{A} 6=119.08344707 \backslash \mathrm{A} 7=122.7295546 \backslash \mathrm{A} 8=120.306329 \backslash \mathrm{A} 9=119.863$ $50317 \backslash \mathrm{A} 10=119.96275832 \backslash \mathrm{A} 11=120.05432501 \backslash \mathrm{A} 12=118.16331091 \backslash \mathrm{A} 13=118.94841$ $311 \backslash \mathrm{A} 14=120.64789891 \backslash \mathrm{A} 15=111.16163895 \backslash \mathrm{A} 16=108.63678332 \backslash \mathrm{A} 17=111.1556043$ $2 \backslash \mathrm{D} 4=-0.00199296 \backslash \mathrm{D} 5=0.0011695 \backslash \mathrm{D} 6=0.00077444 \backslash \mathrm{D} 7=-179.99654983 \backslash \mathrm{D} 8=-180.0$ $0123644 \backslash \mathrm{D} 9=179.99814907 \backslash \mathrm{D} 10=-179.99865381 \backslash \mathrm{D} 11=180.00061604 \backslash \mathrm{D} 12=180.001$ $53654 \backslash \mathrm{D} 13=179.99289489 \backslash \mathrm{D} 14=179.98757396 \backslash \mathrm{D} 15=-59.68188874 \backslash \mathrm{D} 16=180.03271$ $711 \mathrm{D} 17=59.75750313 \|$ Version $=\mathrm{IBM}-\mathrm{RS} 6000-\mathrm{G} 98 \mathrm{RevA} .7 \backslash \mathrm{HF}=-384.8959876 \backslash \mathrm{RMSD}$ $=9.505 \mathrm{e}-09 \backslash \mathrm{RMSF}=1.265 \mathrm{e}-04 \backslash \mathrm{Dipole}=0.2345396,-0.000119,1.1565998 \backslash \mathrm{PG}=\mathrm{C} 01$ [X(C8H8O1)]1\@ 